\title{
Percorsi di integrazione tra auditing e controllo di gestione
}

\author{
Luciano Marchi, Giulio Greco*
}

\begin{abstract}
Integration patterns between auditing and management control

This article reviews the research streams published on this journal. We review the journal integrated research approach within the planning, management and information systems fields. Integrated analysis regards the internal-external dimensions, as well as the public-private dimension of the firm. The article links these themes to the papers included in this issue.
\end{abstract}

Keywords: auditing, management control, performance, private sector, public sector

\section{Analisi revisionale e prospettica}

La rivista Management Control affronta da sempre le tematiche di pianificazione, informazione e controllo, ricorrendo ad un approccio integrato di analisi.

L'integrazione deve essere realizzata sui sistemi di misurazione interna dei costi e delle performance, ma coinvolge anche, necessariamente, la tecnologia informatica, la struttura organizzativa ed i sistemi operativi. L'obiettivo finale è infatti quello di coordinare i sistemi informativo-contabili con quelli di management e governo aziendale. Tale coordinamento può realizzarsi efficacemente con un approccio di analisi integrata, revisionale e prospettica, ai diversi livelli di pianificazione, informazione e controllo.

Ad un primo livello, l'analisi riguarda i comportamenti delle persone, integrando l'analisi revisionale, cioè di conformità dei comportamenti alle re-

\footnotetext{
* Dipartimento di Economia e Management, Università di Pisa.
} 
gole, con l'analisi prospettica rivolta, in particolare, a indirizzare i comportamenti operativi e l'eventuale cambiamento delle regole e/o della loro applicazione.

Ad un secondo livello, l'analisi riguarda la qualità delle informazioni, integrando l'analisi revisionale, cioè il giudizio sull'accuratezza dei dati e la loro coerenza all'interno del sistema informativo, con l'analisi delle prospettive informativo-gestionali, con particolare riferimento alle caratteristiche qualitative delle informazioni richieste ed alle modalità per garantire un efficace utilizzo delle stesse a supporto della gestione aziendale.

Ad un terzo livello, l'analisi riguarda la qualità della decisioni, integrando l'analisi revisionale su efficienza, efficacia ed economicità delle operazioni di gestione con l'analisi delle prospettive sui sistemi di management e controllo-guida della gestione aziendale.

Per ciascuno dei sopra indicati tre livelli di integrazione dell'analisi revisionale e prospettica, il presente numero della rivista fornisce un contributo di ricerca.

Il primo articolo è quello di Rizzotti e Lombardo contenente un'analisi dei fattori influenti sull'efficacia del whistleblowing (segnalazione interna e/o esterna all'azienda di frodi) come strumento di controllo interno. Il lavoro analizza dapprima i fattori che influiscono sull'efficacia degli strumenti di segnalazione dell'esistenza di frodi. Viene poi condotto un esperimento sul contesto italiano, di cui vengono analizzate le caratteristiche culturali in comparazione alla realtà statunitense già documentata in altre ricerche.

Il secondo articolo è riferito a Caldarelli, Ferri e Maffei. Il lavoro affronta il tema dei sistemi informativi aziendali, con un approccio di analisi revisionale e prospettica, osservando i più recenti sviluppi dell'integrazione informativa "interno-esterno": l'utilizzo del cloud computing nelle PMI italiane. L'articolo esamina anche i rischi derivanti dall'utilizzo dei network informatici che consentono l'accesso in remoto ad informazioni condivise localizzate su provider esterni alle aziende.

Nel terzo articolo, curato da Marchi e Bertei, gli autori esplorano i possibili sviluppi legati all'interazione tra performance audit e performance management nel settore pubblico. Da un lato, l'audit può influenzare positivamente la performance e l'accountability in generale. Dall'altro lato, un uso inappropriato può produrre effetti negativi come l'eccessivo focus sulla correttezza della misurazione piuttosto che sugli obiettivi o uno scarso legate tra indicatori di performance e performance stessa. 


\section{Approccio integrato di analisi}

L'approccio integrato di analisi può riguardare anche la gestione costiperformance, cioè il passaggio dalla misurazione dei costi all'analisi, misurazione e gestione integrata costi-performance.

L'approccio integrato di analisi può estendersi, inoltre, alla dimensione pubblico-privato, per indagare sulle uniformità e difformità presenti nei sistemi di governo e controllo gestionale delle aziende pubbliche e private.

L'approccio integrato di analisi può riguardare, infine, l'analisi internoesterno che, a partire dalle misurazioni, si estende alla governance estesa, passando attraverso gli strumenti del controllo relazionale.

Il presente numero della rivista riprende e rilancia proprio alcune delle suddette linee tematiche di ricerca, a partire dall'integrazione costi-performance. Il lavoro di Fornaciari e Marchini, in particolare, studia la gestione dei costi della scarsa qualità (cost of poor quality), analizzando il case study di un'azienda del settore delle acque minerali. Gli Autori presentano un caso di costruzione di un sistema integrato costi-performance ispirato alla crescita sostenibile e in grado di conciliare indicatori di performance economico-finanziari e indicatori sociali e ambientali.

Un ulteriore gruppo di articoli riguarda l'integrazione pubblico-privato su metodologie e strumenti di controllo gestionale. Su tali problematiche sono presenti l'articolo di Gigli, Tieghi e Mariani e quello di Borgonovi, De Matteis e Preite, oltre al già citato lavoro di Bertei che evidenzia potenzialità e limiti dell'applicazione degli strumenti di audit e controllo gestionale delle aziende private in ambito pubblico.

Il lavoro di Gigli, Tieghi e Mariani fornisce una review della letteratura sull'utilizzo dei performance management systems (PMS) nella aziende sanitarie. Alla mappatura della letteratura precedente si affianca l'individuazione di possibili linee di ricerca future. Gli Autori indicano inoltre come possibile obiettivo di politiche sanitarie la promozione di una cultura della valutazione della performance tra gli operatori, con accettazione della stessa a livello di individui nell'ambito dell'organizzazione.

L'articolo di Borgonovi, De Matteis e Preite, si sofferma sulla rilevanza delle spese per organi di governo negli enti locali. Gli Autori studiano gli aspetti di possibile miglioramento dell'efficienza e al possibile connesso recupero di legittimazione politica.

I lavori successivi di Fabietti e Trovarelli e di Albanese toccano il tema dell'integrazione interno-esterno nella prospettiva informativo-contabile. L'integrazione-informativa interna-esterna delle misurazioni è espressione 
dell'evoluzione del controllo di gestione verso la misurazione bilanciata delle performance, verso il monitoraggio ambientale e il controllo strategico.

L'articolo di Fabietti e Trovarelli analizza il ruolo dell'eco-controllo nell'implementazione di modelli di business sostenibili. L'eco-controllo apre a sistemi integrati che usano informazioni economico-finanziarie e ambientali per scopi diversi, tra cui monitorare la compliance con la normativa ambientale, fornire dati per decisioni interne sostenibili e per la rendicontazione esterna.

Chiude il fascicolo l'articolo di Albanese che studia il sistema fieristico italiano alla luce della crisi economica che ha colpito il Paese in anni recenti. Il lavoro esamina empiricamente un campione di fiere negli anni del picco della crisi tra il 2008 e il 2011. I risultati mostrano una sensibile riduzione delle performance economico-finanziarie sia in termini di vendite che di profittabilità, segnalando l'esigenza di un riposizionamento verso l'internazionalizzazione, specie per le realtà di dimensioni più contenute.

\section{References}

D'Onza G., Greco G. \& Allegrini M. (2016), Full cost accounting in the analysis of separated waste collection efficiency: A methodological proposal, Journal of environmental management, 167, pp. 59-65. Doi: 10.1016/j.jenvman.2015.09.002.

Greco G., Ferramosca S. \& Allegrini, M. (2014). Exploring intellectual capital in family firms. An empirical investigation, International Journal of Learning and Intellectual Capital, 11(2), pp. 91-106. Doi: 10.1504/IJLIC.2014.060802.

Greco G., Ferramosca S. \& Allegrini M. (2015), The Influence of Family Ownership on LongLived Asset Write-Offs, Family Business Review, 28(4), pp. 355-371.

Doi: 10.1177/0894486515590017.

Mancini D. (2010), Il sistema di informazione e controllo relazionale per il governo della rete di relazioni collaborative d'azienda, Milano, Giuffrè.

Marchi L. (2011), L'evoluzione del controllo di gestione nella prospettiva informative e gestionale esterna, Management Control, 3, pp. 5-16. Doi: 10.3280/MACO2011-003001.

Marchi L. (2011), Integrazione pubblico-privato su metodologie e strumenti di controllo gestionale, Management Control, 2, pp. 5-8. Doi: 10.3280/MACO2011-002001.

Paolini A. (1993), Il controllo strategico. Uno schema d'analisi, Milano, Giuffrè. 